\title{
Impact of Higher Order Adaptive Filtering for Stereo System Audio Signal
}

\author{
A. A. Amin ${ }^{1}$, M. S. Islam², M. A. Masud ${ }^{3}$, M. N. H. Khan ${ }^{{ }^{4}}$ \\ ${ }^{123}$ Department of Electrical and Electronic Engineering, Uttara University, Dhaka-1230, Bangladesh \\ ${ }^{4}$ School of Electrical, Mechanical and Mechatronic Systems, University of Technology Sydney, \\ 15 Broadway, Ultimo NSW 2007 \\ *Corresponding author, e-mail: nomanxp76@gmail.com
}

\begin{abstract}
In present stereo audio system is a most popular audio system for different purposes. Now a day's stereo system is commonly used in communication and other purposes. Moreover Normalized Least Mean Square (NLMS) based adaptive filtering is an effective filtering process in case of communication and other applications. However adaptive filtering is an adaptive filter process to cancel out the noise from audio signal successfully. Hence the main objective of this paper is to design a NLMS adaptive filter which cancels out the noise from a noisy wave format stereo audio file. Moreover by varying the order of the adaptive filter (such as $8^{\text {th }}, 16^{\text {th }}, 32^{\text {th }}$ and $64^{\text {th }}$ ), the performance of the NMLS adaptive filtered signal with respect to reference and noisy stereo audio signal are analyzed as well.
\end{abstract}

Keywords: Adaptive filtering, Normalized Least Mean Square (NLMS), Stereo system, Least Mean Square (LMS), Filter order, Step size, MATLAB

Copyright $\odot 2017$ Institute of Advanced Engineering and Science. All rights reserved.

\section{Introduction}

The stereo system is most promising audio system in present. It has different channel such as left and right where as both channel have identical audio signal. The audio signal can be distorted by noise or interference [1-2]. So in case of communication and other system distorted signals causes erroneous signal reception which is unwanted in case of any system. However digital filtering has low power consumption and effective result of noise cancellation as well. So the main concern of this paper is to simulate a normalized least mean square (NLMS) adaptive filter for stereo system [3]. As input signal a stereo audio signal will be used. Research background (Segment 2) describes some previous works on adaptive filtering and shows some performance analysis of LMS and NLMS adaptive filtering process for noise cancellation and similar purposes in recent times. Afterwards the result analysis and discussion part illustrates the simulation result analysis of NLMS adaptive filtering of different filter orders. In this paper $8^{\text {th }}$, $16^{\text {th }}, 32^{\text {th }}$ and $64^{\text {th }}$ order filtering process will be used for result analysis for the stereo audio signal. Hence the channels are identical for stereo system so only left channel will be considered for result analysis. Moreover the step size of the adaptive filter is fixed $(\mu=0.03)$ for each case of simulation. In every case the different order adaptive filtered signal will be compared with reference audio signal and the noisy input audio signal.

\section{Research Background}

Adaptive filtering is an efficient and cost effective noise cancellation process for different kind of signals. However in recent times many research works have been done based on adaptive filtering for audio and different kinds of signals. In 2011 Sanjay K. Nagendra and Vinay Kumar S.B. shows some performance evaluation of acoustic noise cancellation of audio signal using LMS algorithm [4]. Furthermore Lilatul Ferdouse et al performed some simulation of adaptive filtering algorithm for noise cancellation [5]. In addition GK Girisha et al also done some performance evaluation of adaptive filtering for hearing aid application in 2015 [6]. Moreover in 2016 Shweta B Thorat et al designed a FPGA based adaptive filtering for audio enhancement [7]. 
The main concern of this paper is to design and simulate of a NLMS adaptive filter with fixed step size. Whereas the order of the adaptive filter will be varying and evaluate the performance of the filtered signal. Moreover the comparative study will be done for stereo signals with respect to reference and noisy signal.

\section{NLMS Adaptive Filter Designing for Stereo System}

A finite impulse response adaptive filter can estimate the desired signal from the provided noisy signal. Figure 1 shows the block diagram of adaptive filtering which explicitly shows how to calculate the error signal and estimate the desired signal as well.

$$
\mathrm{e}(\mathrm{n})=\mathrm{v}_{\mathrm{l}}(\mathrm{n})
$$

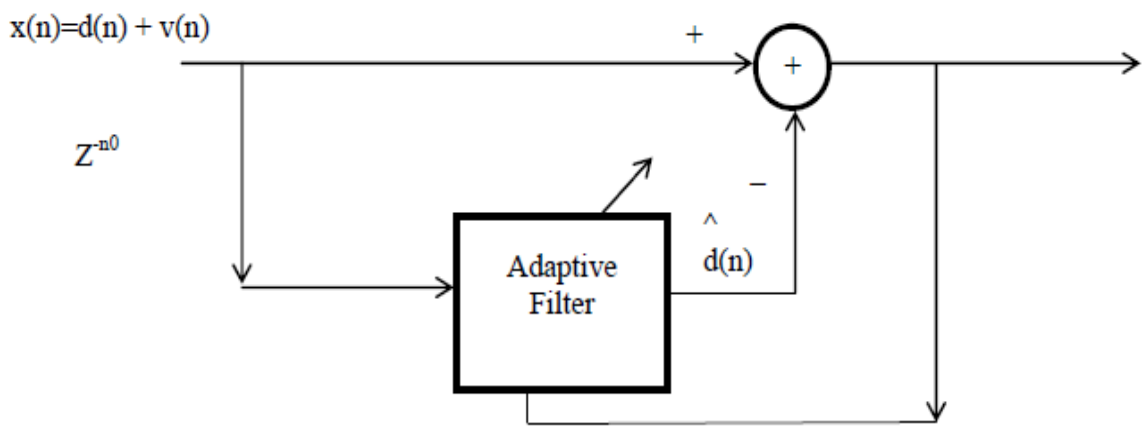

Figure 1. Block diagram of adaptive filtering [8]

Hence Equation 1 \& 2 calculated the desired estimated signal from given signal [8].

$$
\begin{aligned}
& \hat{d}(n)=\sum_{k=0}^{p} w_{n}(k) x(n-k) \\
& \hat{d}(n)=W_{n}^{T} X(n)
\end{aligned}
$$

Where,

$$
\begin{aligned}
& W_{n}{ }^{T}=\left[w_{n}(0), w_{n}(1), w_{n}, . ., w_{n}(p),\right]^{T} \\
& X(n)=[x(n), x(n-1), \ldots, x(n-p)]^{T}
\end{aligned}
$$

In above $W_{n}{ }^{\top}$ represents the transpose of predictor coefficients and $X(n)$ represents the input signal for filtering. So the filter coefficients are calculated by equation 3 and 4 accordingly [8-9].

$$
\begin{aligned}
& \mathrm{W}_{\mathrm{n}+1}=\mathrm{W}_{\mathrm{n}}+\Delta \mathrm{W}_{\mathrm{n}} \\
& \mathrm{W}_{\mathrm{n}+1}=\mathrm{W}_{\mathrm{n}}+\mu \nabla \varepsilon(\mathrm{n})
\end{aligned}
$$

Here, $\Delta W_{n}$ is correction coefficient whereas $\mu$ is step size which should be sufficiently small and $\nabla \varepsilon(\mathrm{n})$ represents the gradient of error. However in case of normalized LMS algorithm the filter coefficients $\left(\mathrm{W}_{\mathrm{n}+1}\right)$ are measured by Equation 5 .

$$
W_{n+1}=W_{n}+\beta \frac{X^{*}(n)}{\|X(n)\|^{2}+\varepsilon} e(n)
$$


Where $e(n)$ is the error, $X(n)$ is the input signal, $X^{*}(n)$ is the conjugate of input signal $\beta$ is normalized stem size for NLMS adaptive filtering and $\varepsilon$ is mean square error. Moreover $\|X(n)\|^{2}$ change the magnitude but the direction remains same as well. At first a noisy stereo audio signal of wave format has been read my MATLAB [10-11]. Afterwards the signal left and right channel signals are split up and filtered based on the NLMS adaptive filtering method to cancel out the noise from the input signal. Figure 2 showed the block diagram of the proposed adaptive filtering noise cancellation system.

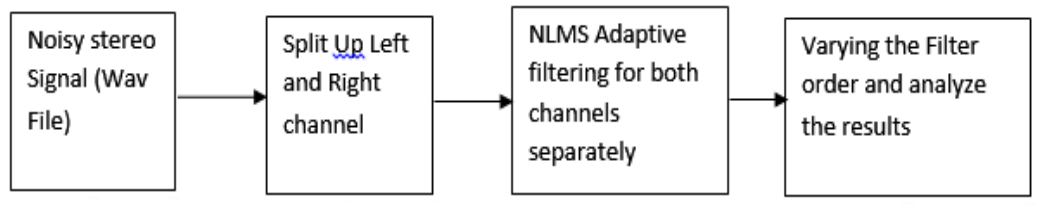

Figure 2. Block diagram of NMLS noise cancellation system for stereo system

Figure 2 illustrates the NMLS noise cancellation systems for stereo audio file. However in this paper the left channel is only considered hence for stereo system both channels are identical [13]. So the NLMS adaptive filtered has been implemented for stereo system and varying the order of the filter to analyze the performance evaluation of the system with respect to noise cancellation [14-15]. The result analyses of different order adaptive filters are discussed explicitly in result and discussion segment.

\section{Results and Analysis}

The setup of the NLMS adaptive filter system has been showed in Figure 2. The whole model is simulated in MATLAB. However as stereo audio a wave file has been read by MATLAB code. In addition the left and right channels are split up and for filtering only left channel has been considered in this paper hence both channels are identical in case of stereo system. Whereas only 1000 samples are considered to get the clear view of input and filtered signals as well. Figure 3 illustrates the impact of $8^{\text {th }}$ order adaptive filtering for the noisy stereo audio signal which is marked by red. Here this is clearly visible that the impact of the noise is bit higher after filtering process, where the noisy signal is marked by green.

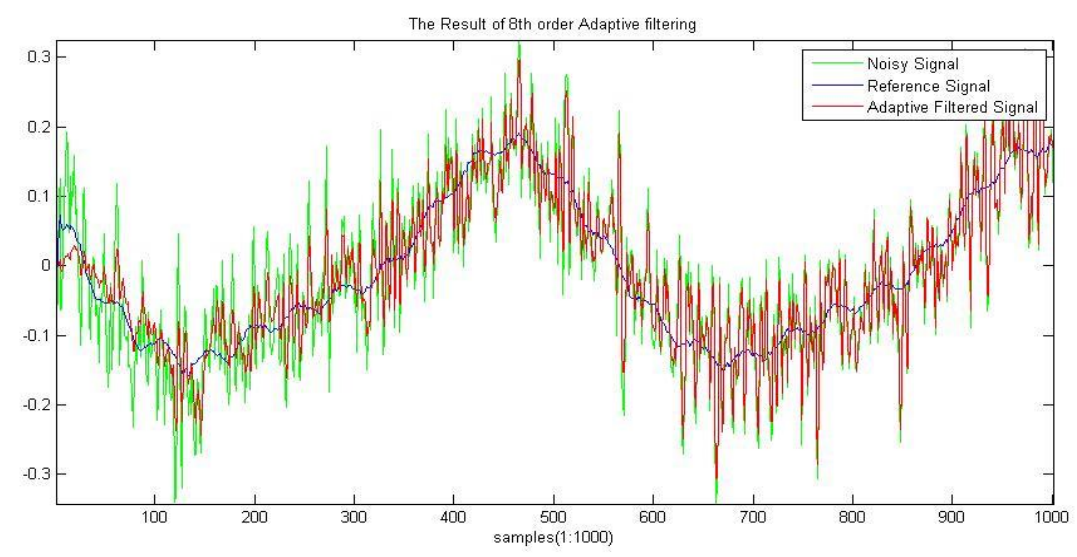

Figure 3. Impact of $8^{\text {th }}$ order NLMS adaptive filtering on noisy stereo audio signal

However it follows the reference audio signal which is marked by blue with higher noise impact. Furthermore Figure 4 illustrates the impact of $16^{\text {th }}$ order filtering on the stereo signal whereas the impact of noise is much lower than $8^{\text {th }}$ order adaptive filtering. 


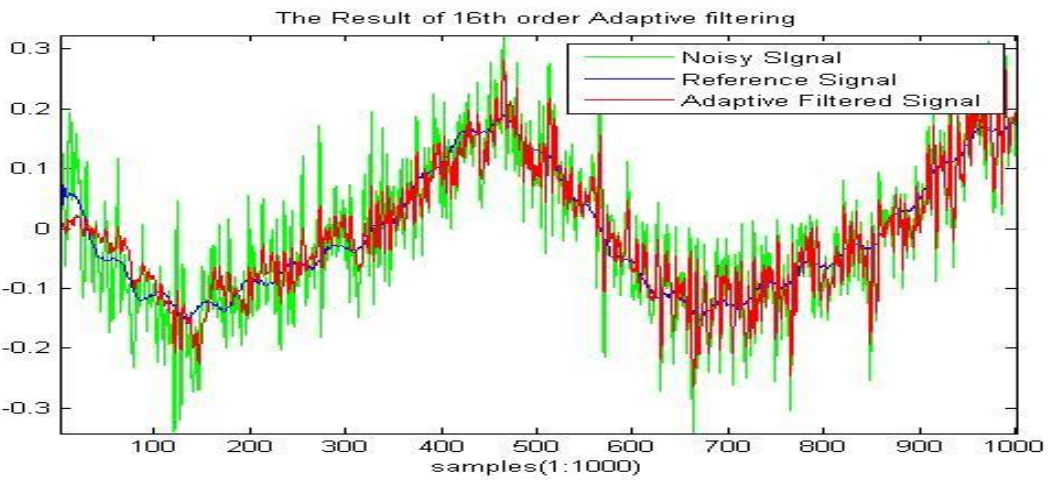

Figure 4. Impact of $16^{\text {th }}$ order NLMS adaptive filtering on noisy stereo audio signal

On the other hand if the order of the NLMS adaptive filtering process is increased more. Suppose $32^{\text {th }}$ order filtering process is implemented then the impact of the noise on the stereo audio signal decreased more which is marked by read on Figure 5. Moreover the NLMS adaptive filtered signal follows the reference audio signal almost same way.

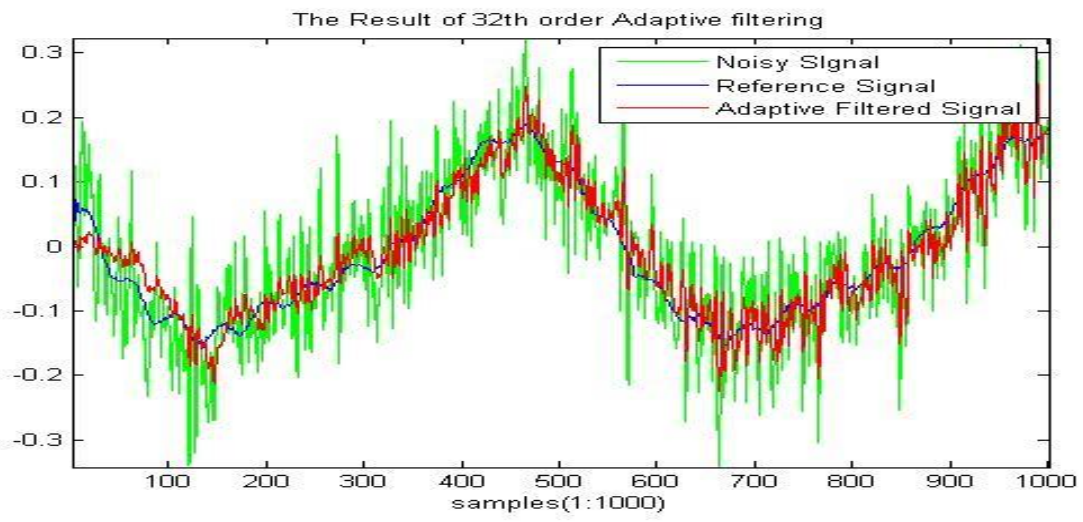

Figure 5. Impact of $32^{\text {th }}$ order NLMS adaptive filtering on noisy stereo audio signal

In addition for higher order such as $64^{\text {th }}$ order NLMS adaptive filtering the filtered signal (Red) is much more identical to reference signal (Blue) as well which is showed in Figure 6.

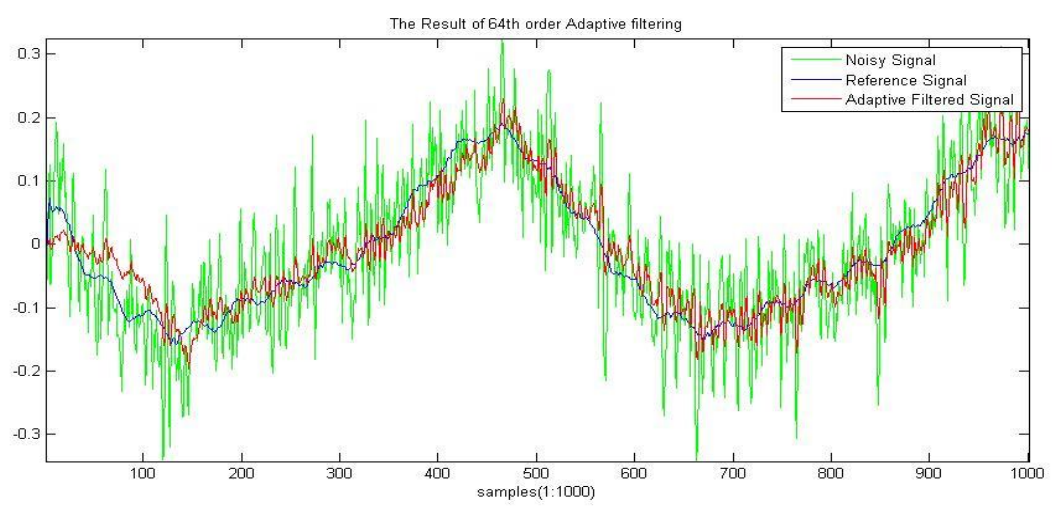

Figure 6. Impact of $64^{\text {th }}$ order adaptive filtering on noisy stereo audio signal 
So it is clearly visible that the higher order NLMS adaptive filtering provides much convincing result than lower order filtering process for stereo system. Moreover the filtered signal is almost identical in case of higher order NLMS adaptive filtering and it also follows the reference audio signal as well.

\section{Conclusion}

Stereo audio signal is very commonly used signal in case of communication and different purposes. However without proper filtering process it is very difficult to recover the original signal for different channels in case of stereo system. Hence in case of stereo system, Normalized Least Mean Square based adaptive filtering is an efficient filtering process for noise cancellation. In contrast the impact of the filter order has been analyzed here for stereo audio signal. In this paper four different order filters such as $8^{\text {th }}, 16^{\text {th }}, 32^{\text {th }}$ and $64^{\text {th }}$ are analyzed for wave formatted stereo audio file. So by result analysis this is clearly visible that for stereo system, the higher order filter (such as $64^{\text {th }}$ order) can cancel out impact of the maximum noise and the $64^{\text {th }}$ order NMLS adaptive filter provides almost identical signal as reference stereo audio signal.

\section{References}

[1] Mahesh S Chavan, Nikos Mastorakis. Studies on Implementation of Haar and Daubechies Wavelet for Denoising of Speech Signal. International Journal of Circuits, Systems and Signal Processing. 2010; 8(3): 83-96.

[2] Syed A Hadei and M loftizad. A Family of Adaptive Filter Algorithms in Noise Cancellation for Speech Enhancement. International Journal of Computer and Electrical Engineering. 2010; 2(2): 307-315.

[3] A Pandey, LD Malviya, Vineet Sharma. Comparative Study of LMS and NLMS Algorithms in Adaptive in Adaptive Equalizer. International Journal of Engineering Research and Applications. 2012; 2(3): 1584-1587.

[4] Sanjay K Nagendra, Vinay Kumar SB. Echo Cancellation in Audio Signal using LMS Algorithm. National Conference on Recent Trends in Engineering \& Technology. 2011; 3 (1): 1-5.

[5] Lilatul Ferdouse, Nasrin Akhter, Tamanna Haque Nipa and Fariha Tasmin Jaigirdar. Simulation and Performance Analysis of Adaptive Filtering Algorithms in Noise Cancellation. International Journal of Computer Science. 2011; 8(1): 185-198.

[6] GK Girisha, SL Pinjare. Performance Analysis of Adaptive Filters for Noise Cancellation in Audio Signal for Hearing Aid Application. International Journal of Science and Research (IJSR). 2016; 5(5): 364-368.

[7] Shweta B Thorat, SINipanikar. Adaptive Filter using FPGA for Audio Enhancement. International Journal of Emerging Technology and Advanced Engineering. 2016; 6(8): 244-248.

[8] Monson H Hayes.Statistical Digital Signal Processing and Modeling. John Wiley and Sons, $3^{\text {rd }}$ edition. 1999.

[9] Simon Haykin, Adaptive Filters Theory, Pearson Education,2008

[10] J Dhiman, S Ahmad and K Gulia. Comparison between Adaptive filter Algorithms (LMS, NLMS, RLS). International Journal of Science, Engineering and Technology Research (IJSETR). 2013; 2(5): 1-5.

[11] A Pandey, LD Malviya, Vineet Sharma. Comparative Study of LMS and NLMS Algorithms in Adaptive in Adaptive Equalizer. International Journal of Engineering Research and Applications. 2012; 2(3): 1584-1587. 